\title{
Global mapping of greenhouse gases and air pollutants
}

Adelbert P.H. Goede', John P. Burrows ${ }^{2}$ and Michael Buchwitz ${ }^{2}$,

${ }^{1}$ FOM Institute Rijnhuizen • the Netherlands • E-mail: A.P.H.Goede@Rijnhuizen.nl

${ }^{2}$ Institute of Environmental Physics (IUP), University of Bremen • Germany

$\mathrm{M}$ an must rise above the earth, to the top of the clouds and beyond, for only thus will he fully understand the world in which he lives, Socrates (470-399 B.C.) observed. Two and a half millennia later satellites orbiting the earth duly obliged, taking images spectrally resolved of the earth atmosphere in order to gain a better understanding of ozone chemistry, air pollution and of climate change.

SCIAMACHY (Scanning Imaging Absorption spectroMeter for Atmospheric CHartograghY) fighting light and shadow in Greek mythology, is an optical spectrometer [1,2,3 and see Box 1] developed by German, Dutch and Belgian scientists and industry as a contribution in kind to ENVISAT, Europe's biggest satellite (see Box 2). Launched on March $1^{\text {st }}$ 2002 by a European Ariane-5 rocket, it has since completed nearly 30.000 orbits around the earth, producing more than 35 million measurement scenes. Its projected 5 year lifetime has now passed. Time to take stock.

\section{Climate change}

A useful concept in climate research is radiative forcing, the change in net irradiance $\left(\mathrm{W} / \mathrm{m}^{2}\right)$ at tropopause height (between 8 and $18 \mathrm{~km}$ ) exerted by a change in greenhouse gas concentration. For small changes this provides a measure for the change in temperature at the earth surface. The human induced effect is of the order of a few $\mathrm{W} / \mathrm{m}^{2}\left(\mathrm{CO}_{2} \sim 1.5 \mathrm{~W} / \mathrm{m}^{2}\right.$, $\mathrm{CH}_{4} \sim 0.7 \mathrm{~W} / \mathrm{m}^{2}, \mathrm{O}_{3} \sim 0.35 \mathrm{~W} / \mathrm{m}^{2}$ ), compared with a globally av- eraged solar irradiation of $340 \mathrm{~W} / \mathrm{m}^{2}$ [4]. Greenhouse gas radiative forcing can be calculated from measurement of the concentration distribution and is most sensitive in the upper part of the troposphere.

The underlying parameter for greenhouse gas concentrations are the sources and sinks of the greenhouse gases. Emissions may be estimated from measured emission factors in combination with statistical data, the so-called bottom-up approach used by countries reporting under the United Framework Convention on Climate Change and the Kyoto Protocol. Emissions can also be derived from measurement of the concentration distribution and inverse modelling, the so-called top-down approach. Discrepancies exist between the two approaches whilst uncertainties are considerable [4]. This limits our understanding of climate change and adversely affects verification of greenhouse gas emission inventories.

Inverse modelling has been carried out from ground based observations, but results are limited by their scarce and unbalanced distribution over the earth surface [5]. From their vantage point in space satellite measurements have the benefit of global coverage, but measurement accuracy in the lowest layers of the atmosphere is a challenge. This challenge was met by EVERGREEN (EnVisat for Environmental Regulation of GREENhouse gases), a European Commission RTD project carried out by a consortium of 12 European organisations [6].

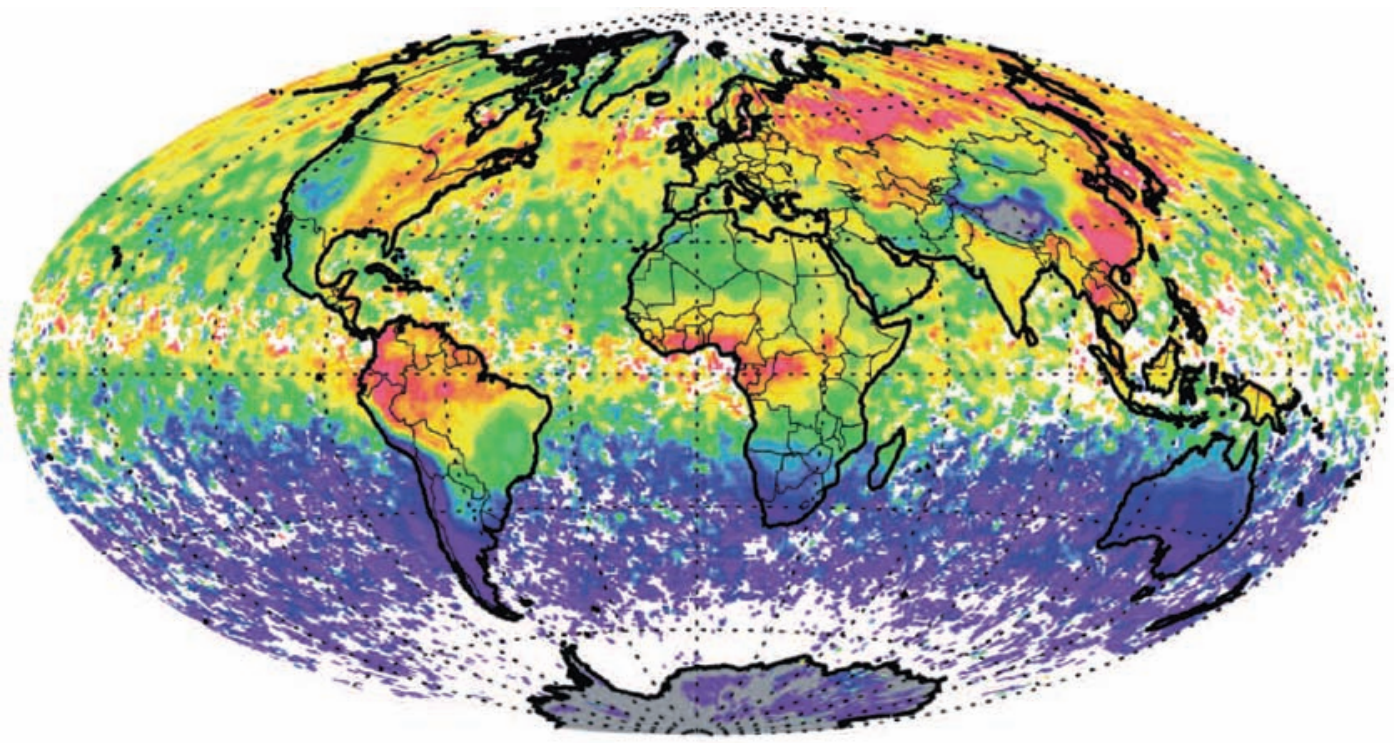

Methane mixing ratio [ppb]
4 Fig. 1: The global distribution of atmospheric methane (column-averaged dry air mixing ratio) retrieved from SCIAMACHY near-infrared nadir spectra over the year 2003. Most methane sources reside in the Northern Hemisphere (wetland, rice paddies, ruminants), which shows up as a North-South hemisphere gradient. Major source regions are clearly visible such as wetlands in Russia, rice paddies and wetlands in China and broad leaved forest areas in South America and wetlands in Central Africa (Schneising et al., 2007, ref. 8) 
This spectrometer combines a large spectral range (240 nm to $2400 \mathrm{~nm})$ with a medium spectral resolution $(\sim 0.2 \mathrm{~nm})$ accomplished in two stages. First the light is pre-dispersed by a prism. Subsequently, the spectrum formed is peeled off and directed into eight separate channels in which final dispersion is obtained by a grating. Thus, high and low signal regimes are separated to suppress stray light and to avoid overlapping spectral orders.

The approach was made possible by the development of solid-state linear array (1024) detectors which measure the entire spectrum in one go, rather then scanning the spectrum over a photo multiplier tube, the common technology at the time (1988). The near infrared (1000-2400 nm) InGaAs detectors were developed and space qualified by the project. The measurements presented in this paper originate with these unique detectors.

The InGaAs photo-sensitive material is epitaxially grown in thin layers of increasing In content such as to reduce the band gap energy and increase the cut-off wavelength to match the channel wavelength range. The lattice mismatch results in dislocations which produce noise. To suppress noise the detectors are passively cooled to $150 \mathrm{~K}$ by a 2 -stage radiator directed into deep space and connected by cryogenic heat pipes to the detectors.

The atmosphere is sampled by a mirror system in front of a telescope collecting the incoming light. A nadir scan provides a $1000 \mathrm{~km}$ wide swath at a horizontal resolution of approx $30 \mathrm{~km}(\mathrm{~N}-\mathrm{S})$ by $60 \mathrm{~km}(\mathrm{E}-\mathrm{W})$. A limb scanning mirror yields vertical information at a resolution of approximately $3 \mathrm{~km}$. Resolution is specific to the trace gas investigated and depends on entrance slit width, detector pixel size, signal to noise performance and data rate. After six days the entire surface of the Earth is sampled, which determines the temporal resolution of the measurements.

The Picture shows one level of the optical instrument, housing the spectral channels 1 and 2, the scan mirrors, the telescope and the calibration unit. The pentagonal element in the centre is the pre-dispersing prism, the lynch pin of the instrument which forms an intermediate spectrum, which subsequently is peeled off and relayed to individual channels. Not shown is the second level of the instrument housing the spectral channels 3 to 8 . Also not shown are the electronic control and data processing units, the thermal control unit and the cooling system.

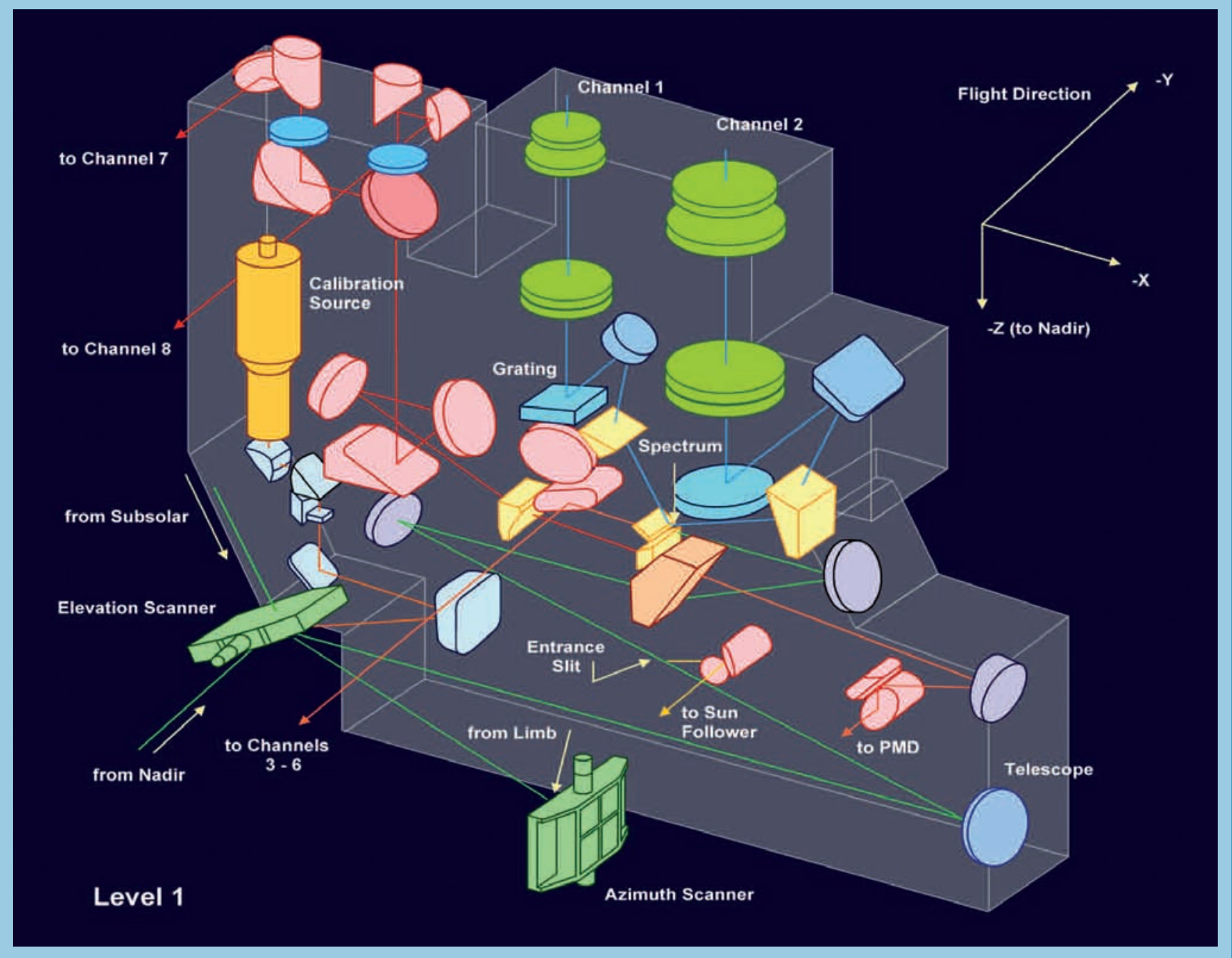



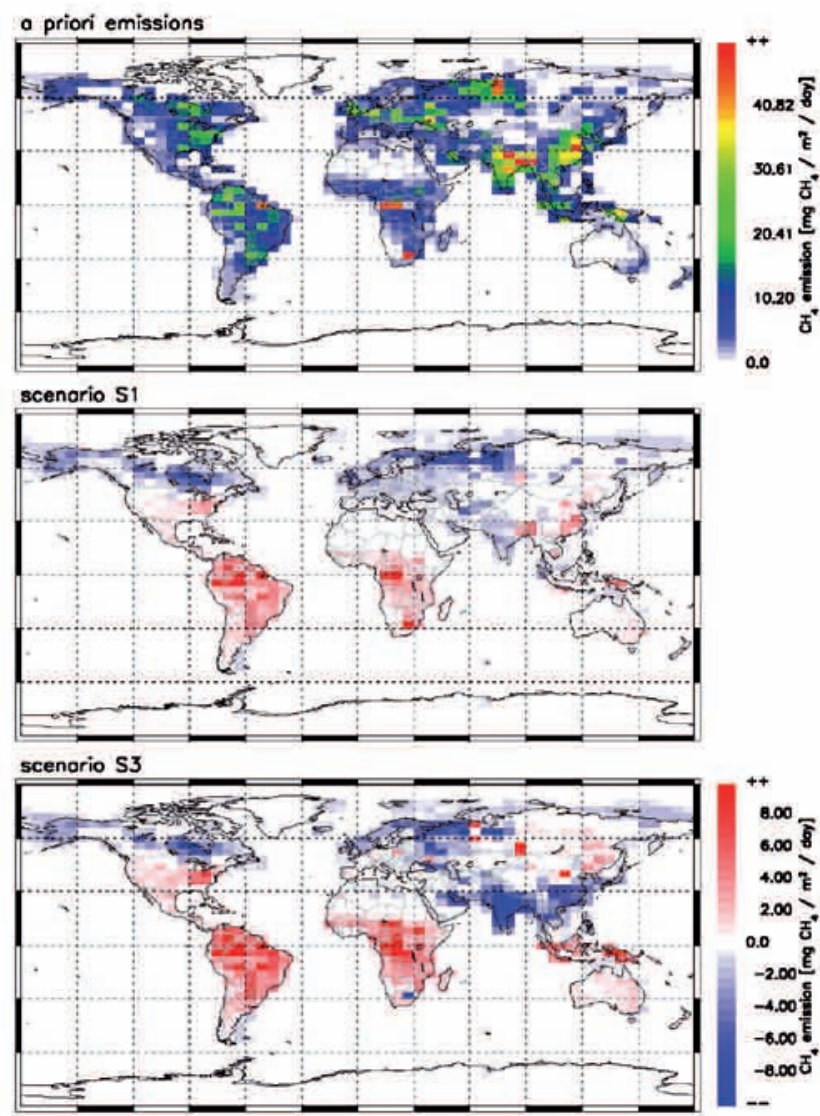

$\Delta$ Fig. 2: Methane source strength (top) is improved by inverse modelling, in scenario S1 with ground based measurements only and in scenario S3 with SCIAMACHY measurements added. Northern Hemispheric sources need to be adjusted downward. Tropical Southern Hemispheric sources need upward adjustment. Unknown sources in the Amazon area and West Africa have been discovered by SCIAMACHY (Bergamaschi et al., 2006, ref 5). Copyright 2007 American Geophysical Union. Reproduced by permission of AGU.

The approach adopted was to use relative measurements (see Box 3). Relative measurements are less prone to errors in instrument calibration and radiation transport calculation. For example, radiation transport can be computed as accurately as one likes, including multiple scattering and polarization; if however the aerosol, haze or (cirrus) cloud distribution is not accurately known, neither will be the scattering path travelled by the photon. One way to overcome this problem is to consider the scattering of a well mixed gas as a proxy for the correction of light path. Using oxygen for the purpose improves precision to a certain extent, but is limited by the fact that the energy of an oxygen photon absorbed at the $\mathrm{O}_{2} \mathrm{~A}$ band $(780 \mathrm{~nm})$ has more than twice the energy of a photon absorbed by methane (1631-1670 nm) or carbon dioxide (1563-1585 nm). Scattering properties of the reference photons are thus different and the correction of the light path not perfect.

For methane this limitation could be avoided by normalizing the measurements on carbon dioxide. The SCIAMACHY results over the period August to November 2003 demonstrate for the first time that methane sources can be detected from space [7]. Compared with theoretical models higher methane abundances are found over tropical areas of South America, Africa and Asia suggesting unknown emissions. Figure 1 shows the global distribution of atmospheric methane for the year 2003 [8].

Methane emissions can be derived from the measured abundances through inverse modelling. Figure 2 shows the first results for the year 2003 based on both ground and satellite data [5]. These results suggest significantly greater tropical emissions compared with bottom-up estimates and compared with inversion based on surface measurements alone. They are attributed to a larger emission from tropical wetlands and termites and a decrease in the soil sink. Global methane emission inventory of $550 \pm 50 \mathrm{Tg} / \mathrm{yr}$ changes little.

Naturally, attention is focused on $\mathrm{CO}_{2}$, the most important anthropogenic greenhouse gas responsible of more than half the anthropogenic radiative forcing. The atmospheric lifetime of $\mathrm{CO}_{2}$ is an order of magnitude larger than that of methane (100 vs.10 years). The disturbance in total column height due to emissions is correspondingly smaller and measurement accuracy will have to be higher. Figure 3 shows the first global $\mathrm{CO}_{2}$ measurements by SCIAMACHY for the years 2003-2005 [9]. These are relative measurements normalized to oxygen, not ideal as explained before. Like methane, comparison with models shows good overall agreement, but also interesting differences. The yearly increase of $\mathrm{CO}_{2}$ is well captured as demonstrated by the 2 in $380 \mathrm{ppm}$ precision. The $\mathrm{CO}_{2}$ sequestration during the growing season and its release during autumn and winter qualitatively agree with ground based measurements and models. Quantitatively however, measurement shows a larger amplitude than modelled. This may be explained by measurement error. It could also point to higher $\mathrm{CO}_{2}$ fluxes on the Northern Hemisphere than hitherto known. This interesting result warrants further investigation.

In order to retrieve emissions from concentration distributions, data assimilation is the appropriate technique. Data assimilation is capable of optimizing a large number of model parameters simultaneously and handling large amounts of measured data. Data assimilation is based on the theorem of Bayes (1763), a Presbyterian priest who mathematically showed how to change religion in the face of new facts. In other words, it is a statistical method that incorporates measurements into a theoretical model to improve its representation of reality. The method is used in meteorology for weather prediction.

Data assimilation allows quantification of information content when adding new measurements to a theoretical model. A sensitivity analysis carried out for methane [10], shows that monthly averaged methane data from SCIAMACHY significantly improve emission data on a sub-continental scale $(\sim 500 \mathrm{~km})$. A necessary condition is to include clouded pixels in order to sufficiently constrain the inversion. This is made possible by the relative measurement approach, where the normalization of methane by carbon dioxide allows clouded pixels to be taken into account. Clouded pixels provide for high reflectivity over the ocean, which is needed for sufficient signal strength of the reflected radiation measured by SCIAMACHY. So far, data assimilation has not been applied to quantify $\mathrm{CO}_{2}$ emissions, being 
limited by measurement error in relation to error in vertical transport modelled.

\section{Air quality}

In the seventies the notion arose that air pollution is not merely a local problem but affects air quality on an (inter)continental scale. Meteorological condition lifts up the polluted air to the free troposphere where long-range transport sets in to subsequently precipitate and raise local air pollution levels more than $1000 \mathrm{~km}$ away. This poses problems to local authorities trying to meet air quality targets. In order to follow and predict air pollution transport the traditional method of air samples taken locally at the ground is not sufficient. Space based instruments add the global dimension. More recently the link to climate change has been established, a warmer climate speeding up chemical reactions and enhancing emissions.

Carbon monoxide (CO) is a toxic air pollutant which plays a role in greenhouse gas chemistry, through the production of troposphere ozone and the destruction of the hydroxyl radical which is a sink for methane. The lifetime of $\mathrm{CO}$ ranges from several weeks to months and therefore $\mathrm{CO}$ is an excellent tracer for atmospheric transport. A large, but poorly known source of $\mathrm{CO}$ is biomass burning, highly variable in space and time. SCIAMACHY retrieves $\mathrm{CO}$ from some weak overtones in the vibration-rotation spectrum at 2.31 to $2.38 \mu \mathrm{m}$. Again, the relative measurement procedure has been applied, now taking methane as the normalization agent. The first results show unprecedented detail in elevated levels of CO, capturing $\mathrm{CO}$ emission of individual cities, see Figure 4 [11]. Inverse modelling of CO sources will be the next step, see [6].

\section{Stratospheric ozone}

Satellite measurements of stratospheric ozone form the most mature part of research. Although reasonably well understood, the difficulty in predicting the trend to recovery and the interaction with climate change have the consequence that continuous monitoring of stratospheric ozone remains mandatory. Ozone also influences the radiation balance and consequently the weather. SCIAMACHY delivers total ozone column data near real time to the European weather centre ECMWF for assimilation in the medium-range weather model.

\section{Validation and application}

The validity of the SCIAMACHY methane and CO measurements has been verified by ground based Infrared Fourier Transform Spectrometry [12]. The accuracy of methane measurements is $1-2 \%$ and of $\mathrm{CO}$ is $10-20 \%$. The accuracy of the $\mathrm{CO}_{2}$ measurements is currently being assessed but is expected to be similar to methane.

The GMES project PROMOTE (PROtocol MOnitoring for The Environment) employs SCIAMACHY data to deliver an operational air quality, ozone and climate service [13]. GMES, Global Monitoring for Environment and Security, is a joint programme of the European Commission and ESA and is the European contribution to the international GEO programme for a global earth observation system addressing a wide range of social and economic applications, such as health, climate and energy.

\section{Conclusion and outlook}

SCIAMACHY has fulfilled its original mission objective to detect greenhouse gasses and air pollutants from space and to improve ozone observations. Over one hundred papers have been published to date in peer reviewed journals including ...

\section{Box 2: Envisat}

The SCIAMACHY instrument is mounted on the top right corner of the satellite, here shown before launch in the ESA clean room (see Picture). In orbit, the satellite moves sideways through space. Dimensions are $26 \times 10 \times 7 \mathrm{~m}$, total mass is $8140 \mathrm{~kg}$ and electrical power is $6.6 \mathrm{~kW}$. Payload mass $2050 \mathrm{~kg}$ comprises 10 instruments. Total cost of the satellite is approximately 2.5 BEuro.

The satellite circles the earth in polar orbit at $800 \mathrm{~km}$ altitude going from North Pole to South Pole and back in approximately 100 minutes. The orbit is synchronised with the position of the sun such as to probe the earth at constant $(10 \mathrm{am})$ local time or constant solar zenith angle. In contrast, a geostationary orbit at $36.000 \mathrm{~km}$ altitude is synchronised with the rotation of the earth sampling a fixed area on earth at changing solar zenith angle.

Data are transmitted at a rate of $100 \mathrm{Mb} / \mathrm{s}$ to European ground stations via the Artemis data relay satellite using the Ka band. Data are also down linked via X-band to the Kiruna ground station at every Arctic overpass. Data collected are stored over one orbit using two $70 \mathrm{~Gb}$ solid state memories and one $30 \mathrm{~Gb}$ tape recorder. Raw data are being processed on ground to calibrated spectra which are subsequently processed to trace gas concentration distributions, see box 3.control unit and the cooling system.

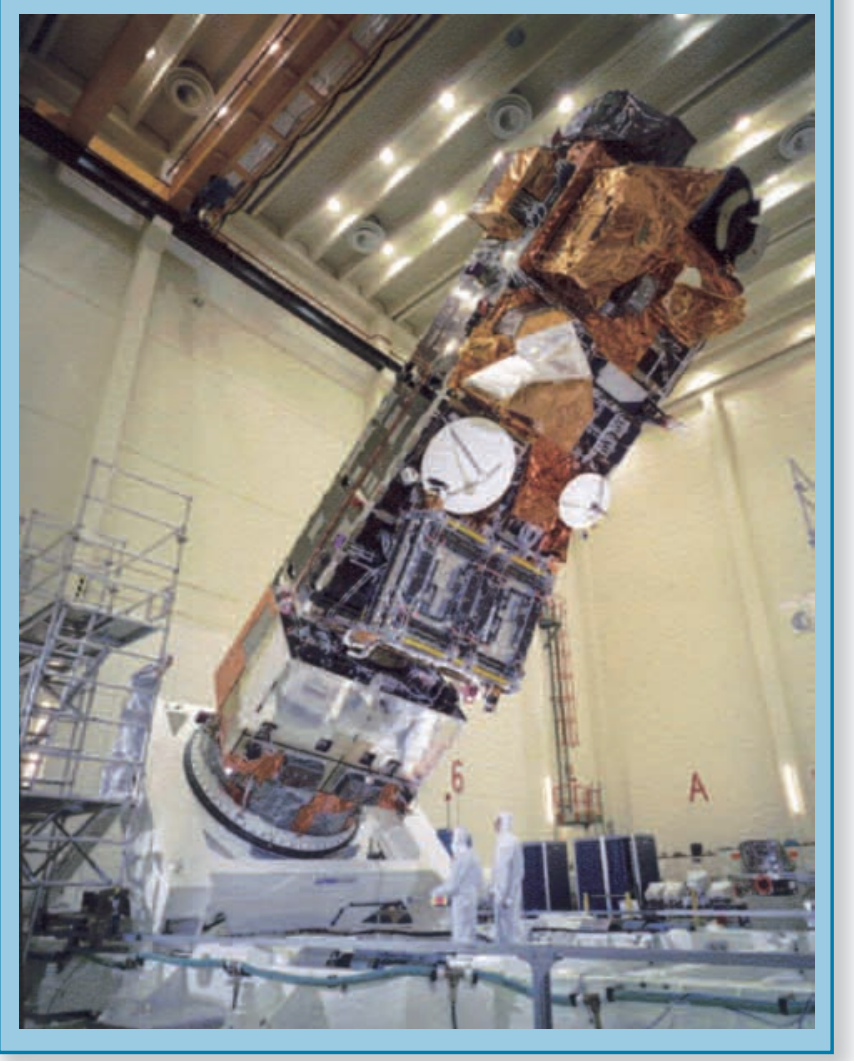




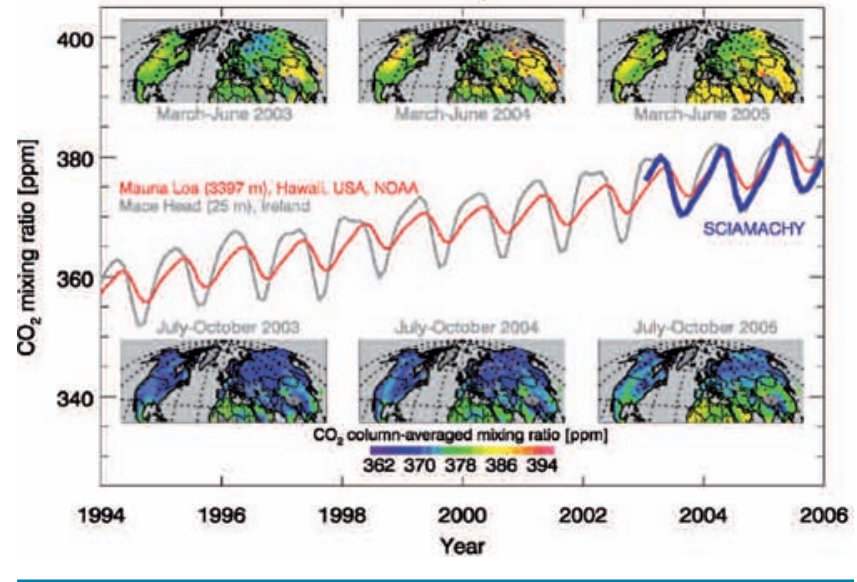

A Fig. 3: Column-averaged mixing ratio of carbon dioxide $\left(\mathrm{CO}_{2}\right)$ during $2003-$ 2005 over the Northern Hemisphere retrieved from SCIAMACHY data. The thin lines indicate local $\mathrm{CO}_{2}$ surface measurements based on weekly flask sampling at Mauna Loa, Hawaii (red line) and Mace Head, Ireland (grey line) by courtesy of T. Conway and colleagues at NOAA, Boulder. The thick blue line shows the SCIAMACHY data averaged over the Northern Hemisphere, displaying our breathing planet, resulting mainly from $\mathrm{CO}_{2}$ uptake and release by growing and decaying vegetation. The annual $\mathrm{CO}_{2}$ increase results mainly from the burning of fossil fuels (Buchwitz et al., 2007, ref. 9).

V Fig. 4: Global distribution of carbon monoxide (CO) during 2004 retrieved from SCIAMACHY measurements. Major CO source regions show up including those in Central Africa (CO from biomass burning during the dry season) and in China (CO from fossil fuel burning). Zooming in reveals elevated $\mathrm{CO}$ at or near major cities (Buchwitz et al., 2007, ref. 11).
... Science and Nature. SCIAMACHY data have found application in the European operational atmosphere service GMES. It has given Europe the lead in atmospheric composition research with improved knowledge of the physics and chemistry of the atmosphere as a result.

The SCIAMACHY mission design lifetime of 5 years has now passed. Extension to 2010 offers some years of grace, but degradation of the instrument has irrevocably set in. The UV channel suffers from cataract whilst the infrared channels lose pixels at an alarming rate, hammered by cosmic rays. In 2010 only $30 \%$ of the pixels will still be alive.

Climate and environmental research needs long term measurement series. The European operational Earth Observation satellite MetOp will extend the SCIAMACHY measurements until 2020 with the instruments GOME-2 and IASI on board. But for innovative scientific satellites no launch opportunity appears to exist before 2018. This is not because of lack of ideas. For example, photo-chemically active climate and environment gases require diurnal time resolution, not days as is currently the case. GeoTrope, a SCIAMACHY derivative in geostationary orbit will provide half hourly synoptic air quality and climate charts over Europe, North Africa, Siberia and part of the Atlantic Ocean.

With climate high on the political agenda and energy the topic of EPS, it is appropriate to see what space based measurements of greenhouse gases can offer in understanding
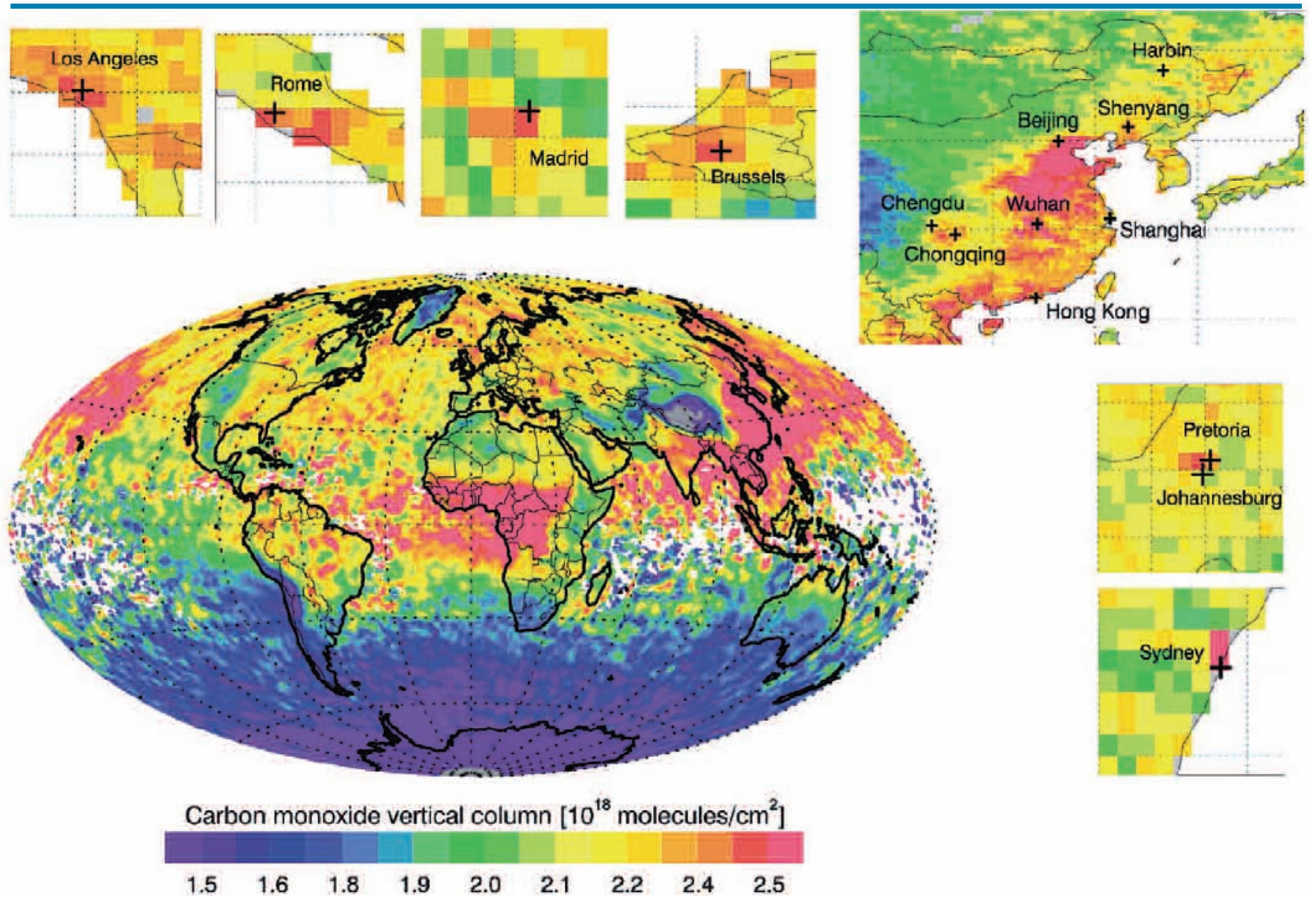
climate change. The EPS will conduct a workshop in collaboration with the German Physical Society on 13 March 2008 inDarmstadt to define the way forward in measuring and modelling the environmental effects of energy production. A series of parallel EPS workshops on novel energy production and conversion physics will culminate in a final workshop organized in collaboration with the French Physical Society later that year. Be alert!

\section{About the authors}

Adelbert Goede initiated atmospheric space research in the Netherlands in 1988 and was SCIAMACHY Co-Principal Investigator until recently. Presently he works at FOM Rijnhuizen (NL) and is Executive Committee member of the European Joint Undertaking for ITER, Barcelona. He is chair of the EPS Environmental Physics Division

John P. Burrows is the Principal Investigator of SCIAMACHY. He is professor of Atmospheric Chemistry and Physics at the University of Bremen and leads there the Institute for Environmental Physics. In 2006 he received the prestigious William Nordberg Medal from the international space research organization COSPAR. He is member of the EPS Environmental Physics Division

Michael Buchwitz is senior scientist at the Institute of Environmental Physics of the University of Bremen. He and his group (including Oliver Schneising, Iryna Khlystova, and Maximilian Reuter) have produced the SCIAMACHY green- house gas and carbon monoxide measurements presented in this paper.

\section{References}

[1] SCIAMACHY Monitoring the changing earth's atmosphere, Editor M. Gottwald, Published by DLR Institut fur Methodik der Fernerkundung, March 2006.

[2] A.P.H. Goede et al., SCIAMACHY Instrument Design, Adv Space Res 11, p.234-236, 1991

[3] H. Bovensmann et al., SCIAMACHY Mission Objectives and Measurement Modes, J. Atmos Sci. 56, pp127-150, 1999

[4] Climate Change 2007-The Physical Science Basis, Working Group I contribution to the Fourth Assessment Report of the Intergovernmental Panel on Climate Change, Cambridge University Press 2007, ISBN13:9780521705967, see http://ipcc-wg1.ucar.edu/index.html

[5] P. Bergamaschi et al. Satellite cartography of atmospheric methane from SCIAMACHY: Evaluation based on inverse model simulations, J. Geophys. Res. 112, D02304 (2006)

[6] A.P.H. Goede et al., EVERGREEN Global satellite observations of greenhouse gases, COSPAR p. A1.1-0207-06 (2006), EGU News Letter 21, September 2007, http://the-eggs.org/articles.php?id=109

[7] C. Frankenberg et al., Assessing methane emissions from global space-borne observations, Science 308 pp. 1010-1014 (2005)

[8] Schneising, O., M. Buchwitz, H. Bovensmann, and J. P. Burrows, Three years of SCIAMACHY carbon dioxide and methane column-averaged dry air mole fraction measurements, Proc. ENVISAT Symp. 2007, ...

Box 3: Data retrivial

SCIAMACHY employs absorption spectroscopy in the near infrared (1000 to $2400 \mathrm{~nm}$ ) to retrieve column abundances of the gases $\mathrm{CO}_{2}, \mathrm{CO}, \mathrm{CH}_{4}$ and $\mathrm{N}_{2} \mathrm{O}$, utilising the sun as the photon source. In contrast, emission spectroscopy in the mid infrared ( 5 to $15 \mu \mathrm{m}$ ) is based on black body radiation. The advantage of near infrared absorption spectroscopy is in the sensitivity to gasses residing in the boundary layer, where thermal infrared spectroscopy lacks contrast with the background black body radiation emitted from the earth surface.

Differential Optical Absorption Spectroscopy (DOAS) exploits the power of relative measurements to gain precision. A known molecular absorption spectrum is matched to a measured spectrum, whilst the adjacent spectral regions yield information on the photon scattering path through the atmosphere. The total column of air mass sampled is converted into vertical columns by a radiation transport calculation. Weighting Function Modified WFM-DOAS takes the height sensitivity of absorbed radiation into account [14]. In a second step, the ratio between the retrieved column and a known gas column $\left(\mathrm{CO}_{2} / \mathrm{O}_{2}, \mathrm{CH}_{4} / \mathrm{CO}_{2}\right.$ or $\left.\mathrm{CO} / \mathrm{CH}_{4}\right)$ further improves precision as residual errors resulting from aerosol and cloud scattering are divided out.

The Figure shows SCIAMACHY sun-normalized radiance spectra measured in nadir geometry. The top panel displays the radiance from channels 1 to 6 covering $240 \mathrm{~nm}$ (ultraviolet) to $1750 \mathrm{~nm}$ (near-infrared). Shaded regions indicate the $\mathrm{O}_{2}, \mathrm{CO}_{2}$, and $\mathrm{CH}_{4}$ column retrieval windows. The middle panels show channels 7 and 8 spectra, the latter being employed for $\mathrm{CO}$ retrieval. The bottom row shows spectral fits, the dots representing the SCIAMACHY measurements and the line representing the fitted radiative transfer calculation..
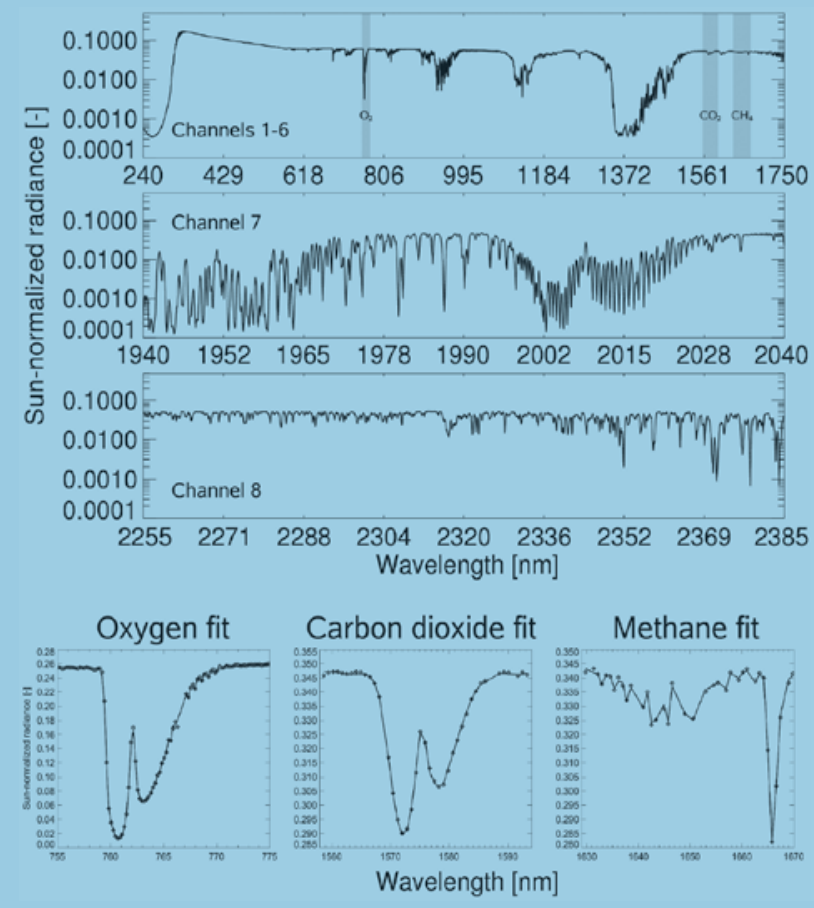
.. Montreux, Switzerland, ESA publications SP-636 (CD), p. 4, 2007.

[9] Buchwitz, M., O. Schneising, J. P. Burrows, H. Bovensmann, M. Reuter, J. Notholt, First direct observation of the atmospheric $\mathrm{CO}_{2}$ year-toyear increase from space, Atmos. Chem. Phys., 7, 4249-4256, 2007.

[10] J-F. Meirink, H. Eskes and A.P.H. Goede, Sensitivity analysis of methane emissions derived from SCAIAMACHY observations through inverse modeling Atmos Chem Phys 6 pp. 1275-1292 (2006)

[11] Buchwitz, M., Khlystova, I., Bovensmann, H., and Burrows, J. P., Three years of global carbon monoxide from SCIAMACHY: Comparison with MOPITT and first results related to detection of enhanced CO over cities, Atmos. Chem. Phys., 7, 2399-2411 (2007)

[12] Dils, B., et al. Validation of WFM-DOAS v0.6 CO and v1.0 CH4 scientific products using European ground-based FTIR measurements,
Proc. 3rd Workshop Atmos. Chem. Validation ENVISAT (ACVE-3), 4-7 Dec. 2006, ESA/ESRIN, Frascati, Italy, ESA Publication SP-642 (CD), 2006, and Dils, B., et al. Comparisons between SCIAMACHY and ground-based FTIR data for total columns of $\mathrm{CO}, \mathrm{CH}_{4}, \mathrm{CO}_{2}$ and $\mathrm{N}_{2} \mathrm{O}$, Atmos. Chem. Phys. 6, 1953-1976 (2006)

[13] A.P.H. Goede et al. PROMOTE Protocol Monitoring for the GMES Service Element, ESA AO/1-4302/02/I-IW, March 2004, see www.gse-promote.org

[14] M. Buchwitz, , V.V. Rozanov, and J.P. Burrows, A near-infrared optimized DOAS method for the fast global retrieval of atmospheric $\mathrm{CH}_{4}, \mathrm{CO}, \mathrm{CO}_{2}, \mathrm{H}_{2} \mathrm{O}$, and $\mathrm{N}_{2} \mathrm{O}$ total column amounts from SCIAMACHY Envisat-1 nadir radiances, J. Geophys. Res. 105, 15,231-15,245, 2000. 